\title{
Cytoplasmic Linker Proteins Regulate Neuronal Polarization through Microtubule and Growth Cone Dynamics
}

\author{
Dorothee Neukirchen and Frank Bradke \\ Max Planck Institute of Neurobiology, Junior Research Group Axonal Growth and Regeneration, 82152 Martinsried, Germany
}

\begin{abstract}
Axon formation is a hallmark of initial neuronal polarization. This process is thought to be regulated by enhanced microtubule stability in the subsequent axon and changes in actin dynamics in the future axonal growth cone. Here, we show that the microtubule end-binding proteins cytoplasmic linker protein (CLIP)-115 and CLIP-170 were enriched in the axonal growth cone and extended into the actin-rich domain of the growth cone. CLIPs were necessary for axon formation and sufficient to induce an axon. The regulation of axonal microtubule stabilization by CLIPs enabled the protrusion of microtubules into the leading edge of the axonal growth cone. Moreover, CLIPs positively regulated growth cone dynamics and restrained actin arc formation, which was necessary for axon growth. In fact, in neurons without CLIP activity, axon formation was restored by actin destabilization or myosin II inhibition. Together, our data suggest that CLIPs enable neuronal polarization by controlling the stabilization of microtubules and growth cone dynamics.
\end{abstract}

\section{Introduction}

The formation of the axon is an initial event during the polarization of neurons (Barnes and Polleux, 2009). It relies on specific cytoskeletal changes that enable the growth of the axon but restrain the growth of future dendrites (Craig and Banker, 1994). Past work showed that the actin cytoskeleton regulates neuronal polarization (Bradke and Dotti, 1999; Kunda et al., 2001). More recently, however, it was revealed that microtubules may also play an active role in the generation of the axon (Conde and Cáceres, 2009). Microtubules of the subsequent axon were found to be more stable than microtubules of future dendrites in cerebellar and hippocampal neurons (Arregui et al., 1991; Witte et al., 2008). Moreover, modest pharmacological stabilization of microtubules transforms both minor neurites and mature dendrites into growing axons (Gomis-Rüth et al., 2008; Witte et al., 2008). While these data suggest that microtubule stability may be a basic process that regulates neuronal polarity, we know relatively little about which molecules regulate neuronal polarization by changing microtubule dynamics (Conde and Cáceres, 2009).

The growth cone, rich in microtubules in its central domain and actin filaments in its periphery, plays a prominent role in the

\footnotetext{
Received July 30, 2010; revised Nov. 10, 2010; accepted Nov. 30, 2010.

This work was supported by the Max Planck Society, the International Foundation for Research in Paraplegia, and additional grants from the Deutsche Forschungsgemeinschaft. D.N. is a recipient of a Boehringer Ingelheim Foundation fellowship. F.B. is a recipient of a Career Development Award from the Human Frontier Science Program. We thank Anna Akhmanova, Claudia Laskowski, Shalin Naik, Michael Sixt, Michael Stiess, and Sabina Tahirovic for critically reading the manuscript. We are indebted to Liane Meyn for technical assistance and for preparing excellent neuronal cultures, Stefanie Kandels-Lewis for providing us with the CLIP antibody, Anna Akhmanova for generously providing us with the used CLIP constructs and pEGFP-EB3, Vic Small for providing us with mCherry-EB3, Eckhard Mandelkow and Jacek Biernat for providing us with the Tau and MAP2c construct, James Chalcroft for writing the program for microtubule growth speed measurements, and Niels Galjart, Ihor Smal, and Erik Meijering for distributing the Kymotracker tool prior to publication.

Correspondence should be addressed to Frank Bradke, Max Planck Institute of Neurobiology, Junior Research Group Axonal Growth and Regeneration, Am Klopferspitz 18, 82152 Martinsried, Germany. E-mail: fbradke@neuro.mpg.de.

D0I:10.1523/JNEUROSCI.3983-10.2011

Copyright $\odot 2011$ the authors $\quad 0270-6474 / 11 / 311528-11 \$ 15.00 / 0$
}

formation of the axon (Witte and Bradke, 2008; Lowery and Van Vactor, 2009). Myosin II-based retrograde transport of actin filaments toward the center of the growth cone results in the generation of actin arcs (Schaefer et al., 2002) that prevent microtubules from invading the peripheral region (Burnette et al., 2008; Lee and Suter, 2008; Schaefer et al., 2008; Lowery and Van Vactor, 2009). During active growth phases, actin arcs reorient toward the growth direction, creating an F-actin-free corridor into which microtubules protrude (Lee and Suter, 2008). Molecules that putatively mediate the interaction between microtubules and actin filaments might enhance this process of polarization (Andersen and Bi, 2000) and, thus, are primary candidates for enabling selective axon growth. Microtubule endbinding proteins are involved in various polarity processes, including migration of neuronal cells (Martínez-López et al., 2005; Bai et al., 2008), axon guidance in invertebrates (Lee et al., 2004), and neurite growth (Stepanova et al., 2003; Shi et al., 2004; Zhou et al., 2004; Geraldo et al., 2008; Muley et al., 2008). We therefore asked whether a microtubule end-binding protein could regulate neuronal polarization.

We focused our investigation on a family of microtubule plusend-tracking proteins ( + TIPs), the cytoplasmic linker proteins (CLIPs)115 and 170, which are both expressed in the developing nervous system (Jaworski et al., 2009). Besides potential interaction of CLIP-170 with the actin cytoskeleton (Fukata et al., 2002), the CLIP-170 homolog Tip1 is a critical regulator of cellular polarity in fission yeast (Brunner and Nurse, 2000).

Here, we show that CLIPs are required for axon formation. Our data indicate that their polarizing activity is mediated by stabilizing the microtubule network in the axon that allows microtubules to protrude to the growth cone leading edge. CLIPs also positively regulate dynamics of the axonal growth cone, whereas CLIP inhibition strongly stimulates actin contractility and arc formation. Thus, CLIPs regulate the polarization of neurons by affecting the microtubule and actin network in the axonal growth cone. 


\section{Materials and Methods}

Cell culture, transfection, and vectors. Primary hippocampal neurons derived from rat embryos were cultured as described previously (Witte et al., 2008). Neurons were transfected before plating with the Nucleofector system (Amaxa) using highly purified DNA (EndoFree Maxiprep; Qiagen) as described previously (Witte et al., 2008). pEGFP-C1 DN170 (amino acids 1027-1320) (Mimori-Kiyosue et al., 2005), pEGFP-C1 DN170 $\Delta$ head (amino acids 348-1390) (Komarova et al., 2002), and pEGFP-C1 MBD170 (amino acids 4-596) based on rat cDNA and pEGFP-EB3 were kindly provided by A. Akhmanova (Erasmus Medical Center, Rotterdam, Netherlands). mCherry-EB3 was a gift from V. Small (Institute of Molecular Biotechnology of the Austrian Academy of Sciences, Vienna, Austria). The constructs pECFP1 hTau40 and pEU-HA MAP2c were kindly provided by E. Mandelkow and J. Biernat (MaxPlanck-Unit for Structural Molecular Biology, Hamburg, Germany). The generation of the Lifeact-RFP plasmid (where RFP is red fluorescent protein) is described (Riedl et al., 2008). For all experiments, pEGFP-N2 (Clontech) was used as control vector. Vectors for expressing small interfering RNA for rat CLIP-115 and rat CLIP-170 were made by inserting DNA oligonucleotides (Metabion) in a plasmid expression vector (RNAi-Ready pSIREN-RetroQ-ZsGreen; BD Biosciences) containing the U6 promoter. The siRNA sequence used for rat CLIP-115 was $5^{\prime}$ ACAAAGCTGAATGGCGGAT-3', and for rat CLIP-170 it was $5^{\prime}$ AGATTACCAGCACGAAATA-3'. For the control vector, the siRNA sequence 5'-GTGCGTTGCTAGTACCAAC-3' was used.

Drug treatment. For long term experiments, $3 \mathrm{~nm}$ taxol (LC Laboratories), $1 \mu \mathrm{m}$ blebbistatin (Sigma-Aldrich), or $1 \mu \mathrm{m}$ cytochalasin D (SigmaAldrich) were added to the culture medium $4-6 \mathrm{~h}$ postplating. Cells were then further incubated at $36.5^{\circ} \mathrm{C}$ in the presence of the drugs and fixed at 2 days in vitro (DIV). For short term experiments, $5 \mu \mathrm{M}$ blebbistatin or 5 $\mu \mathrm{M}$ cytochalasin $\mathrm{D}$ were applied to the neurons on 2 DIV and directly observed by life cell imaging. Drugs were kept as stock solutions in DMSO ( 5 mm taxol, $50 \mathrm{~mm}$ blebbistatin, $10 \mathrm{~mm}$ cytochalasin D).

Live cell imaging and image acquisition. Time-lapse recordings for observing growth cone dynamics, actin retrograde flow, or microtubule growth speed (3-5 s intervals) and single images of living neurons were obtained by mounting the coverslips on HBSS/HEPES-containing closed metal chambers, and images were taken at $37^{\circ} \mathrm{C}$. Images were captured using an Axiovert 135/135TV inverted microscope (Carl Zeiss) and standard filters for green fluorescent protein (GFP), Texas Red, and 4',6diamidino-2-phenylindole using a high performance CCD camera 4912 (Cohu Electronics) and Scion Image Beta 4.0.2 software for Microsoft Windows.

Time-lapse recordings of short term treatments with blebbistatin or cytochalasin D were acquired using a live cell imaging setup (DeltaVision RT; Applied Precision). Microtubule growth was correlated with morphological changes of the growth cones by differential interference contrast (DIC) imaging using the DeltaVision live cell setup.

Immunocytochemistry. To stain for Tau-1, microtubule-associated protein 2 (MAP2), and synapsin-1, cells were fixed with 4\% paraformaldehyde and 4\% sucrose in PBS for 20 min, quenched with $50 \mathrm{~mm}$ ammonium chloride in PBS for 10 min, and extracted with $0.1 \%$ Triton X-100 in PBS for $5 \mathrm{~min}$. To stain for CLIPs, cells were fixed with ice-cold $\mathrm{MeOH}$ for $30 \mathrm{~s}$ followed by $4 \%$ paraformaldehyde and $4 \%$ sucrose in PBS for 20 min, quenched, and extracted as described above. For CLIP and cortactin staining of transfected neurons, the effects of the constructs were assessed by plating the cells after transfection on coverslips with a relocation grid. After image acquisition of GFP-positive cells, the cells were processed as described for the CLIP staining described above. To assess acetylated, tyrosinated, and total tubulin integrated in microtubules without unpolymerized tubulin subunits, cells were simultaneously fixed and permeabilized in PIPES-HEPES-EGTA-magnesium (PHEM) buffer as described by Witte et al. (2008) and quenched as above.

The coverslips were blocked at room temperature for $1 \mathrm{~h}$ in a solution containing $2 \%$ fetal bovine serum (Invitrogen), $2 \%$ bovine serum albumin (Sigma-Aldrich), and $0.2 \%$ fish gelatin (Sigma-Aldrich) in PBS. Cells were then incubated with primary antibodies diluted in $10 \%$ blocking solution. The primary antibodies used were mouse anti-Tau-1 (clone
PC1C6, 1:5000; Millipore), mouse anti-MAP2 (1:5000; Sigma-Aldrich), mouse anti- $\alpha$-tubulin (clone B-5-1-2, 1:20,000; Sigma-Aldrich), mouse anti-acetylated tubulin (clone 6-11-B-1, 1:50,000; Sigma-Aldrich), rat anti-tyrosinated tubulin YL1/2 (1:40,000; Abcam), mouse antisynapsin-1 (1:500; Synaptic Systems), rabbit anti-GFP (1:1000; Research Diagnostic), rabbit anti-CLIP-170 H2 recognizing both CLIPs (1:1000; a kind gift from S. Kandels-Lewis, European Molecular Biology Laboratory, Heidelberg, Germany) (Niethammer et al., 2007), or mouse anticortactin (clone 4F11, 1:500; Millipore). As secondary antibodies, Alexa Fluor 350-, 488-, 555-, or 568-conjugated goat anti-mouse, anti-rabbit, or anti-rat IgG antibodies (1:200 to 1:500; Invitrogen) were used.

Protein extraction and Western blotting. Extracts from cultured hippocampal neurons were prepared by rapidly rinsing the cultures once in room temperature PBS, adding SDS sample preparation buffer (Laemmli, 1970), and boiling for $5 \mathrm{~min}$. Protein concentration was determined applying the Bio-Rad protein assay (Bio-Rad Laboratories) based on the method of Bradford (1976). SDS-PAGE was performed on $8 \%$ polyacrylamide gels and Western blotting was done on polyvinylidene difluoride membranes. Antibodies against rabbit anti-CLIP-170 H2 recognizing both CLIPs (1:5000; provided by S. Kandels-Lewis) and mouse anti-actin (clone AC-40, 1:5000; Sigma-Aldrich) were used.

Image analysis and quantification. Length, area, and intensity measurements were performed using Scion Image Beta 4.0.2 for Microsoft Windows, ImageJ (National Institutes of Health), and MetaMorph (Molecular Devices) analysis software. To analyze movement of fluorescence particles in end-binding product 3 (EB3)-transfected neurons, fluorescence images were acquired in 3-5 s intervals during the experiment. Subsequently, kymographs from the regions of interest were made from the individual images using a purpose-written program. To calculate microtubule growth speeds, the slopes of lines drawn on kymographs from the beginning to the end of individual EB3 movements were measured. Catastrophe frequencies were calculated using Kymotracker (provided by Ihor Small and Erik Meijering, Biomedical Imaging Group, Rotterdam, The Netherlands), a plug-in tool for ImageJ. To quantify the distance of microtubule tips to the leading edge of the growth cone, mCherry-EB3 fluorescence intensity was measured from the tip of the growth cone (visualized by DIC image) up to $20 \mu \mathrm{m}$ along the growth cone using ImageJ analysis software. The ratio of acetylated versus tyrosinated $\alpha$-tubulin was determined from fluorescence intensities of both channels in a square of $3 \times 3$ to $5 \times 5$ pixels in the medial part of each process after background subtraction with Photoshop (Adobe Systems). The ratio of CLIP versus $\alpha$-tubulin was determined from fluorescence intensities of both channels in a square of $5 \times 5$ pixels in the area of highest fluorescence intensity within the growth cones. For CLIP/tubulin ratio quantifications, a threshold of at least 1.2-fold difference of fluorescence intensities between axonal growth cones and the remaining neurites' growth cones was specified. For ratio quantifications in general, axons were defined as a process at least $40 \mu \mathrm{m}$ long.

In all other experiments, axons were identified by Tau- 1 staining. To quantify splayed microtubule length, the distance from microtubule tips to the area where microtubules appeared in a bundled formation was measured. Actin dynamics were quantified for a time period of $50 \mathrm{~s}$ by measuring changes of growth cone area every $10 \mathrm{~s}$. Growth cones were outlined manually using MetaMorph software. Growth cone outlines were pasted onto the following images, and changes of growth cone area between the single images were added together for a total growth cone area change over time. For analyzing actin retrograde flow, images of Lifeact-transfected neurons were taken every $5 \mathrm{~s}$ during the experiment. Retrograde flow was measured as the slope of diagonal lines in kymographs acquired by MetaMorph software.

Statistical analysis. Experimental values are presented as a mean \pm SEM. of the indicated number of determinations. Statistical significance ( $p$ value) was calculated using Student's $t$ test and, when noted, Hampel outlier test.

\section{Results}

CLIPs enrich in the axonal growth cone

We first examined the localization of CLIPs during neuronal polarization. Immunohistochemistry showed that CLIPs exhibit 
comet-like structures in the cell body, the neurites, and their tips (Fig. $1 A, B$ ), resembling the CLIP structures at microtubule tips that have been described in non-neuronal cells (Pierre et al., 1992; Hoogenraad et al., 2000; Komarova et al., 2002; Binker et al., 2007). In the growth cone, CLIPs partially overlapped with the actin cytoskeleton within the transitional zone (Fig. 1A). To assess CLIP distribution in growth cones of axons versus growth cones of minor neurites, we stained cells against CLIPs together with tubulin as internal control. We found that CLIPs were enriched in the growth cone of the axon compared with the minor neurites' growth cones in $82 \pm 3 \%$ of polarized neurons $(n>150$ neurons from three independent experiments for each data point; $p<0.001$ ) (Fig. $1 B$ ). On average from all stage 3 neurons, the axonal growth cone showed a $1.5 \pm 0.4$-fold increase in the fluorescence intensity ratio of CLIP/tubulin staining compared with the growth cones of minor neurites (Fig. $1 D)$. In $33 \pm 4 \%$ of all stage 2 neurons, one growth cone stood out (Fig. $1 B c$ ) and exhibited a significantly higher ratio of CLIP/tubulin staining compared with the mean of the neurites' growth cones $(n>$ 200 neurons from three independent experiments for each data point; $p<0.05$ by Hampel outlier test). On average from all stage 2 neurons, the ratio of CLIP staining versus tubulin staining was increased $1.4 \pm 0.1$-fold in the growth cone of the minor neurite with the highest ratio compared with the average of the remaining neurites' growth cones (Fig. 1C), which may suggest that CLIP enrichment in one growth cone out of several neurites' growth cones could precede axon formation in morphologically unpolarized cells. Collectively, these data show that CLIPs are enriched in the axonal growth cone of stage 3 neurons and the growth cone of one neurite in a subpopulation of stage 2 neurons.

\section{Interference with CLIP function inhibits axon development}

To assess the role of CLIPs in axon development, we interfered with their function in developing neurons. As a first approach, we downregulated CLIPs by transfecting neurons with small hairpin RNA (shRNA) for CLIP-115, CLIP-170, or both. Western blot analysis showed that neurons transfected with CLIP-115/170 shRNA still contained CLIPs after 3 DIV, but they were significantly downregulated after 5 DIV (supplemental Fig. S1 A, available at www.jneurosci.org as supplemental material). This downregulation led to a $56 \%$ reduction in axon growth from 3 to 5 DIV (supplemental Fig. S1B) compared with control cells or neurons transfected with shRNA for either CLIP-115 or CLIP170 alone (supplemental Fig. S1B) (data not shown for single shRNA transfection). This effect was specific to CLIP function, as reintroducing the N-terminal microtubule-binding head domain (MBD) of CLIPs (MBD-CLIP), which is sufficient for microtubule attachment and for increasing microtubule rescue fre- quency rates in Chinese hamster ovary $(\mathrm{CHO})$ cells (Komarova et al., 2002), compensated the axon growth defect in CLIP-115/170 shRNA-expressing neurons (supplemental Fig. S1C).

Downregulation of the CLIP proteins was not achieved during initial neuronal polarization, presumably because of shRNA expression timing or the half-life of CLIP proteins. Thus, to establish whether CLIPs play a role during this process, we interfered with endogenous CLIP function by expressing a dominant-negative (DN) mutant of CLIP-170 (DN-CLIP) (Mimori-Kiyosue et al., 2005). This mutant makes use of the autoinhibition of CLIPs mediated by the intramolecular interaction of the $\mathrm{N}$ and $\mathrm{C}$ termini (Lansbergen et al., 2004). As the DN-CLIP-mutant is comprised of the C terminus of CLIP-170 (Fig. 2A), which enables its binding to the $\mathrm{N}$ terminus of CLIPs, it prevents the binding of endogenous CLIPs to microtubules (Lansbergen et al., 2004).

Consistent with previous studies in fibroblasts (Komarova et al., 2002; Mimori-Kiyosue et al., 2005), we found that expression of DN-CLIP removed endogenous CLIP comets from the microtubules in $56 \pm 10 \%$ of the neurons (Fig. $2 B, C$ ). Assessment of axon formation in DN-CLIP-transfected neurons after 2 DIV showed that only $33 \pm 4 \%$ of these neurons exhibited a Tau-1positive axon, a 50\% reduction compared with GFP-transfected 

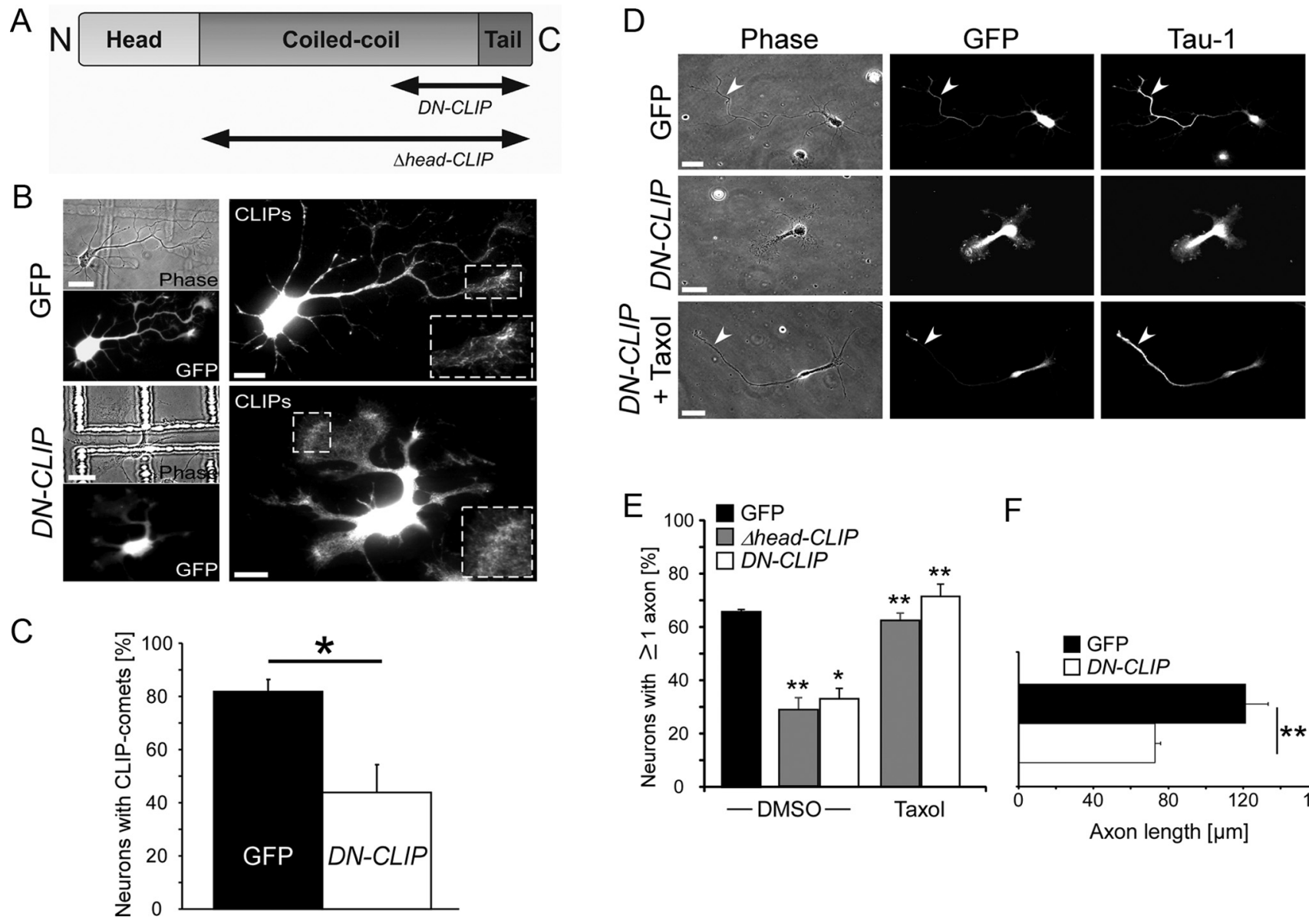

$\mathrm{E}$

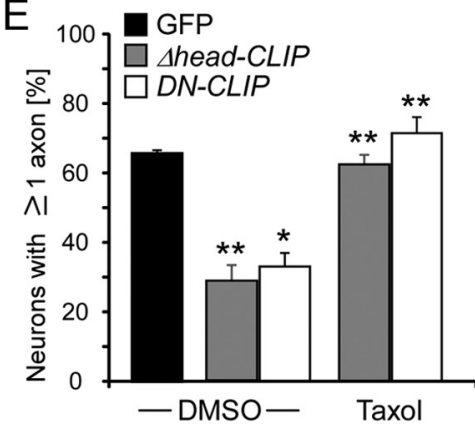

$\mathrm{F}$

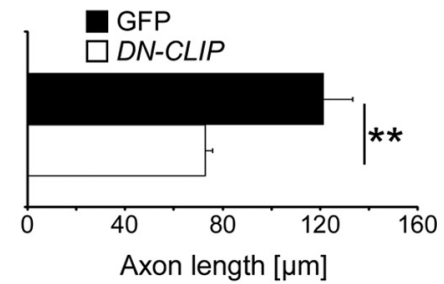

Figure 2. Interference with the function of CLIPs impairs axon development. A, Scheme of the two dominant-negative CLIP constructs, DN-CLIP and $\triangle$ head-CLIP. B, Neurons were transfected with GFP or GFP-tagged DN-CLIP, fixed at 2 DIV, and stained for CLIPs. Enlarged images show higher magnifications of the regions marked in $A$. DN-CLIP-transfected neurons do not show the typical comet-like CLIP-staining. C, Quantification of GFP-transfected (black bar) or DN-CLIP-transfected (white bar) neurons showing regular CLIP comets ( $n>167$ cells from four independent cultures for each data point; $\left.{ }^{*} p<0.05\right)$. D, Neurons were transfected with GFP or a GFP-tagged dominant-negative form of (LIP, DN-CLIP), treated with DMSO or $3 \mathrm{~nm}$ taxol, fixed at 2 DIV, and stained for the axonal marker Tau-1. In DN-CLIP-transfected neurons, axon formation is impaired but is rescued by taxol treatment. Arrowheads indicate axons. Scale bar: $20 \mu \mathrm{m}$. $\boldsymbol{E}$, Quantification of GFPtransfected (black bar), $\Delta$ head-CLIP-transfected (gray bars), or DN-CLIP-transfected (white bars) neurons having $\geq 1$ Tau-1-positive process in cultures treated with DMS0 or taxol ( $n>281$ cells from three independent cultures for each data point, ${ }^{*} p<0.05,{ }^{* *} p<0.01$ ). $F$, Quantification of length of the longest process in neurons transfected with GFP (black bar) or DN-CLIP (white bar) that were fixed at 2 DIV $\left(n>331\right.$ cells from six independent experiments for each data point; $\left.{ }^{* *} p<0.01\right)$.

control neurons (Fig. 2D,E). In addition, the length of axons was drastically reduced (Fig. $2 F$ ). Similar results were obtained when another DN-CLIP mutant, $\Delta$ head-CLIP, which only lacks the microtubule-binding $\mathrm{N}$ terminus, was overexpressed (Komarova et al., 2002) (Fig. 2E). Of note, reintroducing the microtubulebinding domain of CLIPs in DN-CLIP transfected neurons rescued axon formation (supplemental Fig. S2 A, available at www. jneurosci.org as supplemental material). Thus, CLIPs enable initial neuronal polarization and axon formation.

In $D N$-CLIP-transfected neurons, we found a decrease in the ratio of acetylated versus tyrosinated tubulin of the axonal microtubules, which may indicate that these microtubules were less stable compared with the axonal microtubules of control cells (supplemental Fig. S3 $A, B$, available at www.jneurosci.org as supplemental material) (Westermann and Weber, 2003; Witte et al., 2008). Moreover, CLIPs increase microtubule stability in macrophages (Binker et al., 2007) and act as microtubule rescue factors in CHO cells (Komarova et al., 2002). To test whether the loss of axon formation was linked to a loss in microtubule stability, we treated DN-CLIP and $\Delta$ head-CLIP transfected neurons with low doses of taxol (Witte et al., 2008) to modestly stabilize microtubules and monitor any rescue of axon formation. After $24 \mathrm{~h}$ of taxol treatment, $72 \pm 4 \%(D N-C L I P)$ or $62 \pm 3 \%($ head-CLIP $)$ of transfected neurons formed at least one process that was positive for the axonal marker Tau-1, reaching a level of axonbearing cells similar to that of control cells (Fig. 2 D, E; $66 \pm 1 \%$ of $\geq 1$ Tau-1-positive process).

If CLIPs mediate microtubule stability during axon formation, we assumed that other microtubule stabilizers could counterbalance the effect of DN-CLIP. We therefore overexpressed microtubule-associated proteins in DN-CLIP-transfected neurons. Overexpression of Tau or the microtubule-associated protein MAP2c, both of which promote microtubule stability in neuronal and non-neuronal cells (Takemura et al., 1992; Gamblin et al., 1996; Matenia and Mandelkow, 2009), increased axon formation in DN-CLIP-transfected neurons from $30 \pm 3 \%$ to $\sim 50 \%$ (supplemental Fig. S2 B). Interestingly, overexpression of a full-length construct of another + TIP, the end-binding protein EB3, did not rescue axon formation of $D N$-CLIP-transfected neurons (supplemental Fig. S2C). Together, our data show that CLIPs are necessary for axon growth and that they enable neuronal polarization by affecting microtubule stability. 


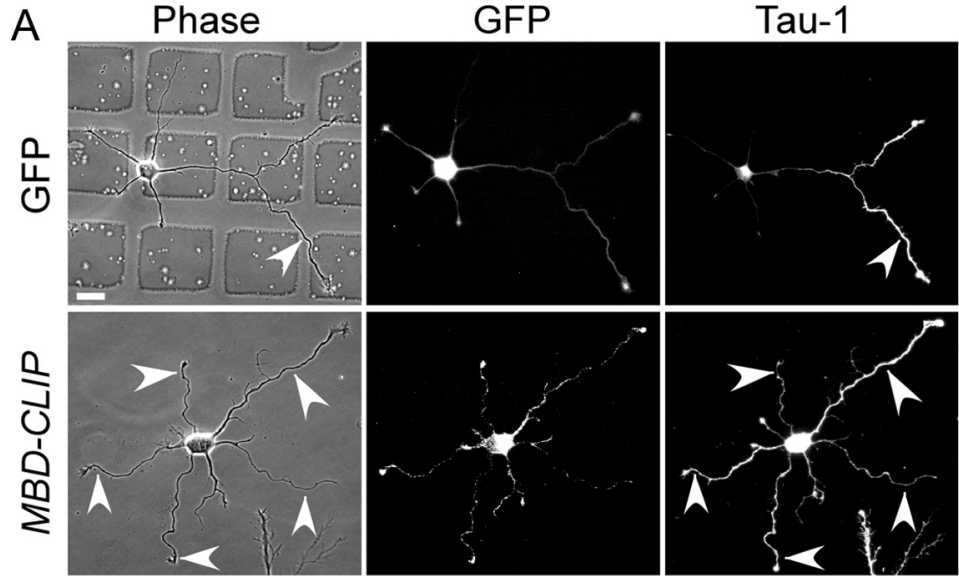

D MBD-CLIP: GFP/Synapsin-1/MAP2

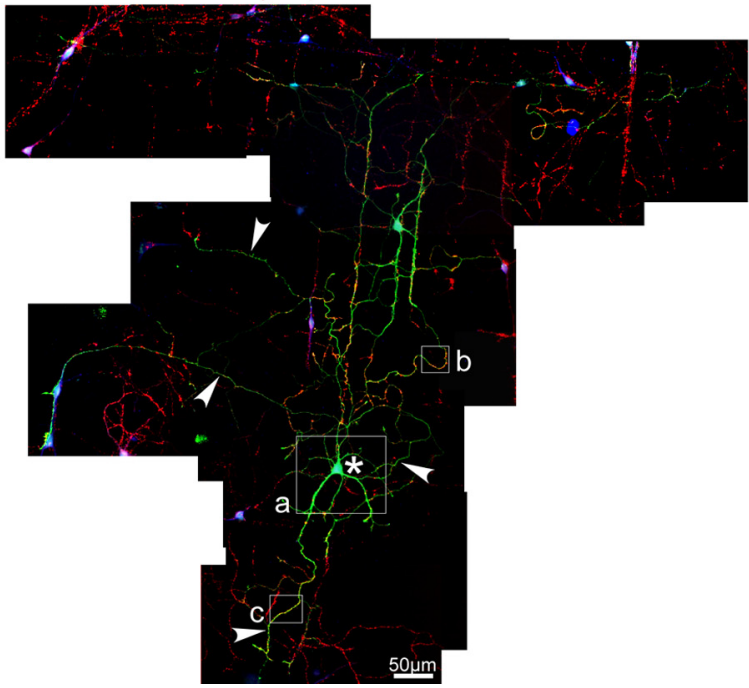

$\mathrm{B}$

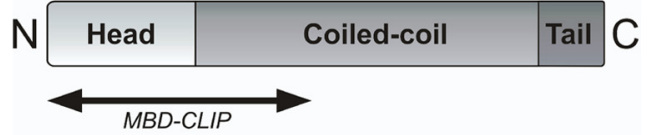

C

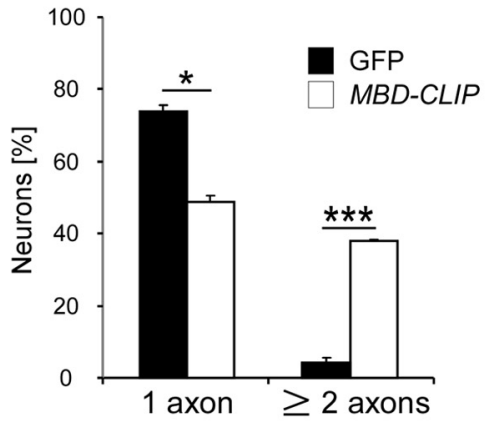

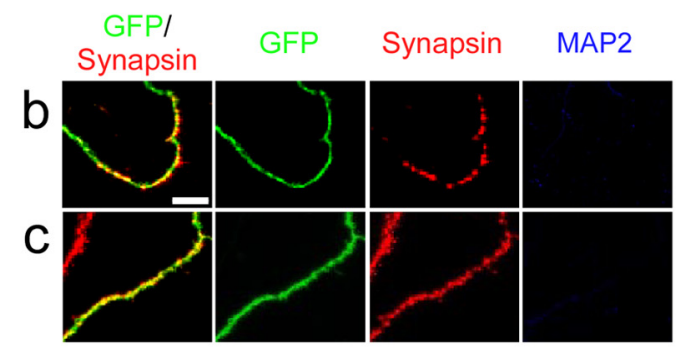

Figure 3. Overexpression of the microtubule-binding domain of CLIPS (MBD-CLIP) leads to the formation of multiple mature axons. A, Neurons were transfected with either GFP or GFP-tagged MBD-CLIP, fixed at 2 DIV, and stained for the axonal marker Tau-1. Scale bar: $20 \mu \mathrm{m}$. Arrowheads indicate axons. B, Scheme of the microtubule-binding domain construct MBD-CLIP. C, Quantification of GFP-transfected (black bars) or MBD-CLIP-transfected (white bars) neurons having one (left) and multiple (right) Tau-1-positive processes ( $n>247$ cells from three independent cultures for each data point; $\left.{ }^{*} p<0.05,{ }^{* * *} p<0.001\right)$. D, MBD-CLIP-transfected neurons at 11 DIV stained for GFP, Synapsin, and MAP2. Asterisk labels a GFP-positive neuron; arrowheads indicate axons. Scale bar: $50 \mu \mathrm{m} . \boldsymbol{a}-\boldsymbol{c}$, Enlarged images of regions marked in $\boldsymbol{C}$. Scale bars: $20 \mu \mathrm{m}(\boldsymbol{a})$ and $10 \mu \mathrm{m}(\boldsymbol{b}, \boldsymbol{c})$.

\section{Overexpression of the microtubule-binding domain of} CLIP170, MBD-CLIP, leads to the formation of multiple axons

If CLIPs have an important role in neuronal polarization, we hypothesized that they may have the potential to turn immature minor neurites, which would later differentiate into dendrites, into axons. Hence, CLIPs may be sufficient to induce axon formation. To test this hypothesis, we overexpressed MBD-CLIP in neurons (Fig. $3 B$ ). We found that $M B D$-CLIP-transfected neurons showed a ninefold increase in the formation of multiple axons (from $4 \pm 2 \%$ in control cells to $38 \pm 2 \%$ in $M B D$-CLIPtransfected neurons) (Fig. $3 \mathrm{~A}, \mathrm{C}$ ). Moreover, $M B D$-CLIP showed an enrichment within the growth cones of the most elongated processes compared with the growth cones of short neurites (supplemental Movie 1, available at www.jneurosci.org as supplemental material). The microtubules of MBD-CLIP transfected neurons displayed the same acetylation-tyrosination ratio as axons of control neurons, suggesting similar microtubule stability (supplemental Fig. S4 A, B, available at www.jneurosci.org as supplemental material). Moreover, the processes of MBD-CLIPoverexpressing neurons were also negative for the dendritic marker MAP2 and positive for the synaptic vesicle protein synapsin-1, showing that the MBD-CLIP processes developed into differentiated axons (Fig. 3D). Thus, overexpression of the microtubule-binding domain of CLIPs is sufficient to induce axon formation.

CLIPs enable dynamic microtubules to protrude into the peripheral domain of the growth cone

As CLIPs changed microtubule stability in the shaft of neurites and were enriched in axonal growth cones, we hypothesized that CLIPs also organize the microtubule network in neurite tips. As previous work suggested that CLIPs mediate bundling of microtubules (Gupta et al., 2009), we analyzed bundling of microtubules at the tip of the neurons in DN-CLIP and MBD-CLIP transfected neurons. In control neurons, microtubules of minor neurites and axons of stage 2 and stage 3 neurons were bundled and reached into the tips of the processes (Fig. $4 A, B$ ). In contrast, microtubules of $D N$-CLIP-transfected neurons failed to coalesce within all processes of both developmental stages (Fig. 4C,D). In axons, the length of splayed microtubules increased from $9.7 \pm$ $0.5 \mu \mathrm{m}$ in GFP-transfected control neurons to $21.7 \pm 0.4 \mu \mathrm{m}$ in 


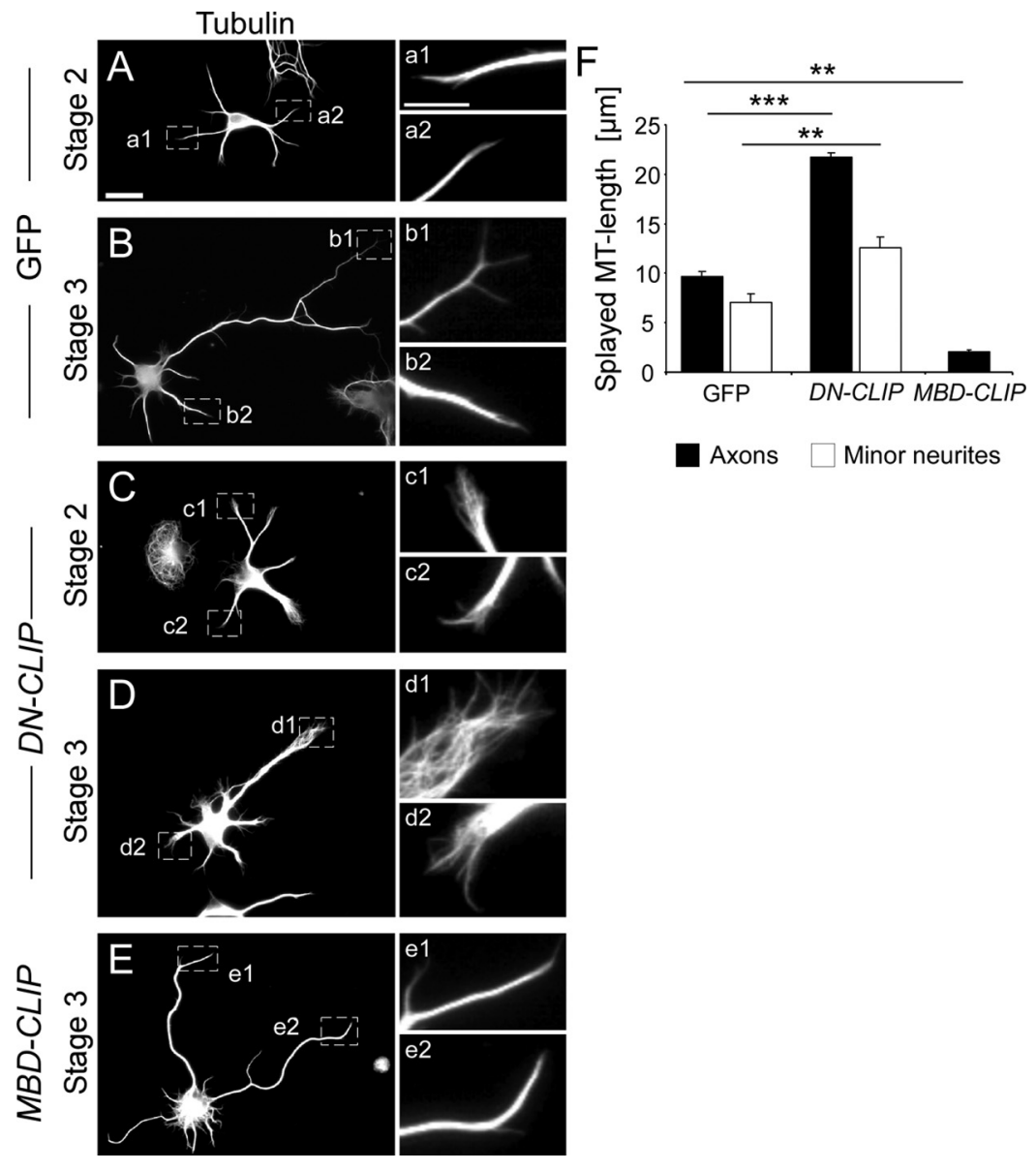

Figure 4. Microtubule structure in DN-CLIP-transfected or MBD-CLIP-transfected neurons. $\boldsymbol{A}-\boldsymbol{E}$, Neurons were transfected with GFP $(\boldsymbol{A}, \boldsymbol{B})$, GFP-tagged DN-CLIP $(\boldsymbol{C}, \boldsymbol{D})$, or GFP-tagged MBD-CLIP $(\boldsymbol{E})$, fixed at 2 DIV, and stained for $\alpha$-tubulin. Microtubules of $D N$-CLIP-transfected neurons are splayed out at the process tips, both in axons and in minor neurites of stage $2(\boldsymbol{C})$ and stage $3(\boldsymbol{D})$ neurons. MBD-CLIP-transfected neurons contain strongly bundled microtubules $(\boldsymbol{E})$. Enlarged images $(\boldsymbol{a} 1-\boldsymbol{e} 2)$ show regions marked in $\boldsymbol{A}-\boldsymbol{E}$. Scale bars $(A-E), 20 \mu \mathrm{m}$; Enlarged images, $10 \mu \mathrm{m}$. $\boldsymbol{F}$, Quantification of splayed microtubule length in axons (black bars) and minor neurites (white bars) of GFP-transfected, DN-CLIP-transfected, or MBD-CLIP-transfected neurons. Splayed microtubule length was defined as distance from microtubule tip to area of bundled microtubules $(n>160$ cells from three independent cultures for each data point; $\left.{ }^{* *} p<0.01,{ }^{* *} p<0.001\right)$.

DN-CLIP transfected neurons (Fig. $4 F$ ). The length of segments with splayed microtubules in minor neurites also increased from $7.0 \pm 0.9 \mu \mathrm{m}(\mathrm{GFP})$ to $12.5 \pm 1.1 \mu \mathrm{m}(D N-C L I P)$. By contrast, $M B D$-CLIP-transfected neurons exhibited strongly bundled microtubules at the tips of all processes $(2 \pm 0.2 \mu \mathrm{m}$ length of displayed microtubules) (Fig. 4E,F).

Development of the growth cone depends on microtubule organization and is therefore potentially regulated by CLIPs in the polarizing neuron. To test whether changes of the microtubule network affect the protrusion of microtubules within the growth cone, we studied the growth rates of microtubules in control as well as in DN-CLIP- and MBD-CLIP-transfected neurons. As EB3 binding to microtubules is independent of CLIP localization and is not affected by DN-CLIP expression (data not shown) (Komarova et al., 2002; Goodson et al., 2003; Bieling et al., 2008), we visualized polymerizing microtubule ends by mCherry-EB3, a well established marker to examine microtubule dynamics (Stepanova et al., 2003; Hasaka et al., 2004).

In GFP alone, DN-CLIP-transfected, or MBD-CLIP-transfected neurons, EB3 comets moved with the same speed along the entire shaft of the axon, indicating that microtubules grew with similar rates $(125 \pm 4 \mathrm{~nm} / \mathrm{s}$ for GFP-transfected neurons, $125 \pm 4 \mathrm{~nm} / \mathrm{s}$ for
DN-CLIP-transfected neurons, and $120 \pm 5$ $\mathrm{nm} / \mathrm{s}$ for $M B D$-CLIP-transfected neurons) (Fig. 5A, $B$ ). Moreover, the number of microtubule catastrophe events remained unchanged between neurons expressing $D N$ CLIP and control neurons $(0.027 \pm 0.001$ events/s in control neurons and $0.030 \pm$ 0.001 events/s in DN-CLIP transfected neurons; $n>128$ microtubules from at least 22 neurons per condition). However, differences in microtubule dynamics were visible in the growth cones. In control conditions and in neurons transfected with $M B D$ CLIP, microtubule plus ends moved into the growth cone toward the leading edge (Fig. $5 C$ ). Around $60 \%$ of their relative EB3 fluorescence intensity was located within a distance of $0.6 \mu \mathrm{m}$ (MBD-CLIP) and 1.0 $\mu \mathrm{m}$ (GFP) from the growth cone tip. By contrast, in DN-CLIP-transfected neurons $60 \%$ of the relative EB3 fluorescence intensity was only found within a distance of $>4.7 \mu \mathrm{m}$ to the membrane edge of the growth cones (Fig. 5C,D). Thus, CLIPs support growth of microtubules into the peripheral domain of the growth cone.

CLIPs regulate growth cone dynamics Because CLIPs regulated microtubules in the growth cone, we wondered whether they also influence growth cone structure and dynamics, processes that affect axon growth. We found that growth cones of $D N$-CLIP-transfected neurons were enlarged from $90 \pm 19 \mu \mathrm{m}^{2}$ in control cells to $224 \pm 33 \mu \mathrm{m}^{2}$, a 2.5 -fold increase (supplemental Fig. S5 $A, B$, available at www. jneurosci.org as supplemental material). Phase contrast live imaging showed that axonal growth cones of GFP or MBD$C L I P$-transfected neurons were highly dynamic and motile (Fig. $6 A, B$ ). In contrast, growth cones of $D N$ $C L I P$-transfected neurons showed very little dynamics (Fig. $6 A, B)$.

To depict the underlying CLIP-mediated changes in growth cone dynamics, we studied actin dynamics using Lifeact-RFP as live marker for the actin cytoskeleton (Riedl et al., 2008). Although the overall growth cone dynamics were significantly impaired after removing CLIPs from the microtubules, the actin retrograde flow speed was not altered in DN-CLIP compared with GFP-overexpressing control cells $(0.13 \pm 0.02 \mu \mathrm{m} / \mathrm{s}$ for $D N-C L I P$ and $0.11 \pm 0.01 \mu \mathrm{m} / \mathrm{s}$ for GFP transfected neurons) (Fig. 6E,F). However, in DN-CLIP-expressing neurons, actin arcs, which are known to prevent microtubule protrusion in the peripheral growth cone (Burnette et al., 2008; Lee and Suter, 2008; Lowery and Van Vactor, 2009), surrounded the central domain of the growth cone perpendicular to the filopodia projection axis (Fig. 6D; supplemental Movie 3, available at www. jneurosci.org as supplemental material). By contrast, in control neurons (Fig. 6C) or neurons expressing MBD-CLIP (data not shown) these arcs were not found. Instead, the bases of filopodia reached close to the central, microtubule-rich domain of the growth cone (Fig. 6C; supplemental Movie 2, available at www. 
jneurosci.org as supplemental material). Hence, we conclude that CLIPs affect growth cone dynamics and that loss of CLIP function induces actin arcs in growth cones.

\section{Manipulation of the actin cytoskeleton rescues axon formation in \\ $D N$-CLIP-transfected neurons}

Actin arcs prevent microtubules from protruding to the peripheral region of the growth cone by myosin II-mediated contractility (Burnette et al., 2008; Lee and Suter, 2008; Schaefer et al., 2008; Lowery and Van Vactor, 2009). We therefore hypothesized that a disassembly of the actin cytoskeleton or an inhibition of the myosin II-mediated actin arc compression could also cause microtubules to protrude further distally into the growth cone, even if CLIPs are nonfunctional. Hence, actin manipulation may be able to rescue axon formation in DN-CLIP-expressing neurons. To test this, DN-CLIP-expressing neurons cotransfected with mCherry-EB3 were treated with cytochalasin $\mathrm{D}$, an actin-depolymerizing drug that converts, in wild-type neurons, nongrowing neurites into growing axons (Bradke and Dotti, 1999, 2000; Kunda et al., 2001). Immediately after applying cytochalasin D to the cells, growth cone dynamics ceased and the actin cytoskeleton depolymerized (data not shown). Subsequently, thin processes containing EB3 comets emerged out of the former growth cone and continued growing (Fig. 7A; supplemental Movie 4 , available at www.jneurosci.org as supplemental material). Similarly, when we applied the myosin II inhibitor blebbistatin to DN-CLIP-expressing neurons, EB3comets reached the growth cone tip and neurites started to grow (Fig. 7B; supplemental Movie 5, available at www. jneurosci.org as supplemental material). Assessment of axon formation after 2 DIV showed that DN-CLIP-expressing neurons formed at least one Tau-1positive axon in $71 \pm 7 \%$ of transfected neurons treated with cytochalasin D and $74 \pm 11 \%$ of transfected neurons treated with blebbistatin (Fig. 7C,D).

Thus, the DN-CLIP-mediated loss of protrusive microtubule force can be counteracted by disassembling the actin cytoskeleton of the growth cone or by releasing the myosin II-mediated compression of actin arcs.

\section{Discussion}

Cytoskeletal dynamics emerged as a key process during neuronal polarization (Arimura and Kaibuchi, 2007; Witte and Bradke, 2008). It was shown that the actin cytoskeleton regulates neuronal polarization (Bradke and Dotti, 1999; Kunda et al., 2001). DN-CLIP.
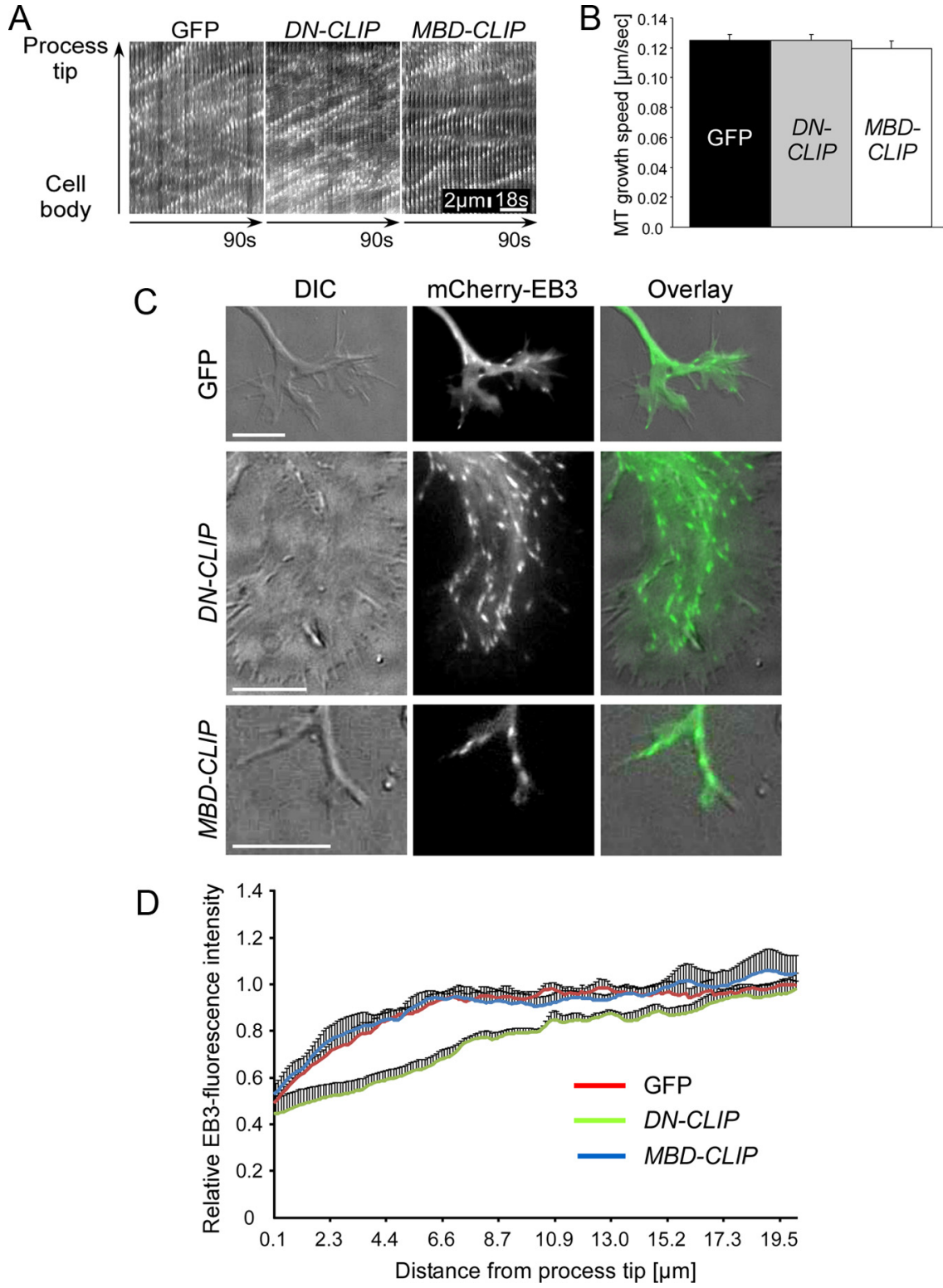

Figure 5. Overexpression of DN-CLIP causes a loss of microtubule ends in the growth cones. Neurons were double-transfected with GFP, GFP-tagged DN-CLIP, or GFP-tagged MBD-CLIP and mCherry-EB3 to visualize microtubule-growing ends. Living neurons were imaged at 2 DIV. A, Kymographs of single processes of neurons transfected with GFP, DN-CLIP, or MBD-CLIP together with mCherry-EB3. Scale bar: $2 \mu \mathrm{m} ; 18 \mathrm{~s}$. B, Quantification of microtubule growth speed in neurons transfected with GFP (black bar), DN-CLIP (gray bar), or MBD-CLIP (white bar) ( $n>61$ EB3-particles from at least 10 neurons). $C$, Images of growth cones of neurons transfected with GFP, GFP-tagged DN-CLIP, or GFP-tagged MBD-CLIP and mCherry-EB3. Microtubule plus ends of GFP-transfected or MBD-CLIP-transfected neurons reach close to the leading edge, whereas in DN-CLIP-transfected neurons microtubules reside in the central domain of the growth cone. Cytoskeletal dynamics were correlated with morphological changes by DIC imaging. Scale bars: $20 \mu \mathrm{m}$. D. Quantification of relative EB3-fluorescence intensity in neurons transfected with GFP (red), DN-CLIP (green), or $M B D-C L I P$ (blue), together with $m$ Cherry-EB3 ( $n>41$ neurons for each data point). Sixty percent of relative EB-3 fluorescence intensity was located within $0.6 \mu \mathrm{m}$ (MBD-CLIP) and $1.0 \mu \mathrm{m}$ (GFP) from the growth cone tip, in contrast to a $4.7 \mu \mathrm{m}$ distance in

Important actin-regulating factors in neuronal polarization and neurite outgrowth include cofilin, Nap-1, and the Ena/Vasp family of proteins (Dent et al., 2007; Garvalov et al., 2007; Jacobs et al., 2007; Yokota et al., 2007). Pharmacological studies depicted microtubule stability as a putative determining factor for neuronal polarization (Witte et al., 2008). As only modest but not strong stabilization of microtubules leads to axon formation (Witte et al., 2008), we sought a microtubule-binding factor that moderately changes the stability of microtubules by affecting 
A
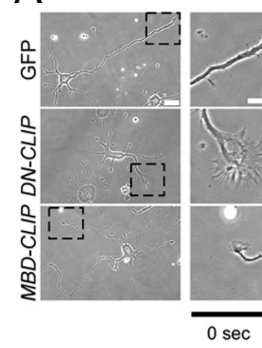
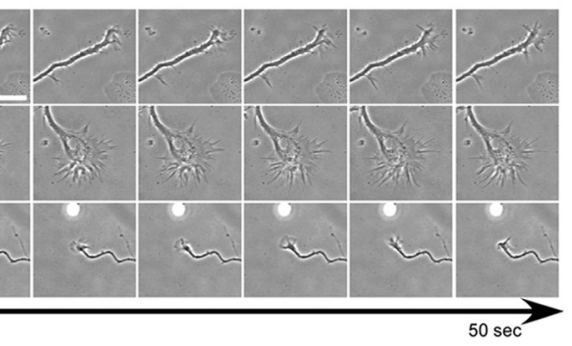

B

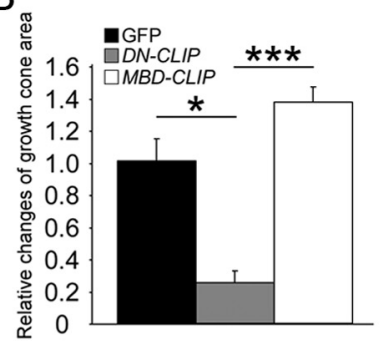

$\mathrm{F}$
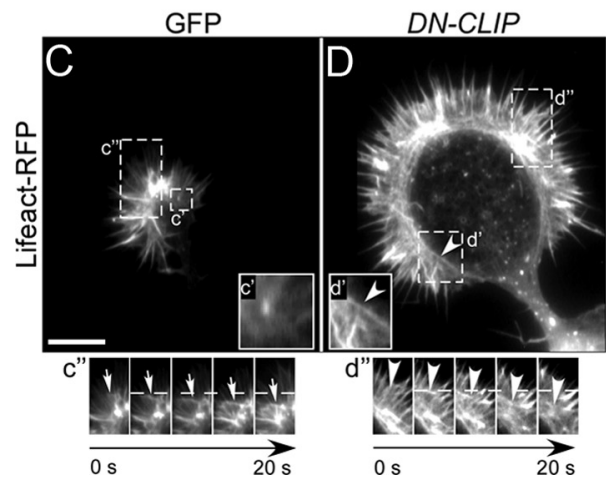
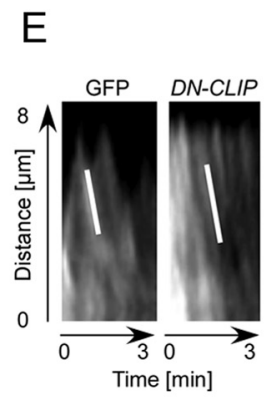

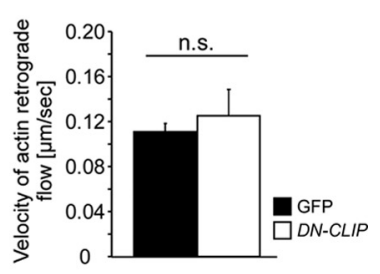

Figure 6. Overexpression of DN-CLIP decreases growth cone dynamics but does not alter actin retrograde flow. $A$, Neurons were transfected with GFP, GFP-tagged DN-CLIP, or GFP-tagged MBD-CLIP and imaged at 2 DIV using live microscopy. Time series of growth cone dynamics of marked areas is shown. Scale bar: $20 \mu \mathrm{m}$. $B$, Quantification of relative changes in growth cone area of GFP-transfected, DN-CLIP-transfected, or MBD-CLIP-transfected neurons ( $n=25$ cells from three independent cultures for each data point; ${ }^{*} p<0.05$, $\left.{ }^{* * *} p<0.001\right)$. C, D, Neurons were transfected with GFP $(\boldsymbol{C})$ or GFP-tagged DN-CLIP $(\boldsymbol{D})$ together with Lifeact-RFP. Living neurons were imaged at 2 DIV. Scale bar: $10 \mu \mathrm{m} . \boldsymbol{c}^{\prime}$ and $\boldsymbol{d}^{\prime}$, Higher magnifications of marked regions of growth cones in $\boldsymbol{C}$ and $\boldsymbol{D}$, showing actin arcs (arrowhead) in DN-CLIP-transfected neurons $\left(\boldsymbol{d}^{\prime}\right) . \boldsymbol{c}^{\prime \prime}, \boldsymbol{d}^{\prime \prime}$, Time series of actin retrograde flow of marked areas in $\boldsymbol{C}$ and $\boldsymbol{D}$. $\boldsymbol{E}$, Kymographs of actin retrograde flow for GFP-transfected and DN-CLIP-transfected neurons. F, Quantification of actin retrograde flow velocity of GFP-transfected (black bar) and DN-CLIP-transfected (white bar) neurons ( $n>26$ cells from three independent cultures for each data point; $p=0.5 ;$ n.s., not significant).

their dynamics. We found that loss of CLIP function inhibited axon formation by decreasing microtubule stability, while overexpression of the microtubule-binding domain of CLIPs enhanced microtubule stability and induced the growth of multiple axons per neuron.

While our data argue that CLIPs enable neuronal polarization by enhancing microtubule stability in the axon, we envision that also other microtubule-regulating proteins may orchestrate axon formation (Witte and Bradke, 2008). These molecules could mediate microtubule stabilization through other molecular mechanisms, including inhibition of microtubule severance or enhancement of microtubule polymerization. The fundamental criterion, however, is that these putative molecules need to modestly enhance microtubule stability in the axon, as this is critical for its growth (Dehmelt et al., 2003; Witte et al., 2008). Consistent with this view, we found that axon formation could be induced in $D N$-CLIP-transfected neurons by overexpression of Tau or MAP2c.

In the case of CLIPs, moderate microtubule stabilization is mediated by their action as microtubule rescue factors (Komarova et al., 2002). This stabilization supported microtubule growth, so that polymerizing ends were enriched in the axonal growth cone. Besides increasing microtubule stability during neuronal polarization, we found that CLIPs also affected growth cone dynamics and the actin cytoskeleton. In particular, CLIPs positively regulated growth cone dynamics and restrained actin arc formation in the transition zone of the growth cone. It was hypothesized that protruding microtubules within the growth cone may function as a "pushing force" for the axon to grow, whereas the actin cytoskeleton may restrict the growth of the protruding microtubules (Burnette et al., 2008; Lee and Suter,
2008; Witte and Bradke, 2008). In support of this hypothesis, we found that depleting the actin cytoskeleton or inhibiting the actin arc contractility in DN-CLIP-transfected neurons enabled microtubules to protrude into the peripheral zone of the growth cone. This protrusion resulted in axon formation. Thus, even less organized microtubules have the ability to induce axonal growth if the opposing actin filaments are lacking or the actin contractility is inhibited.

For the extension, the axonal growth cone repeatedly undergoes a cycle of specific steps (Goldberg and Burmeister, 1986; Lowery and Van Vactor, 2009) (Fig. 8). First, filopodia and lamellipodia, actin-based structures at the growth cone periphery, explore the cellular environment and protrude; the growth cone frequently expands at this stage. Then, dynamic microtubules engorge from the central growth cone area into the former transitional domain, in which actin filaments are disassembled; the remaining actin arcs reorient toward the growth axis. The protruding microtubules transport vesicles, organelles, and, probably, actin-modulating proteins into the engorged area. Interestingly, CLIP-170 was shown to interact with endocytotic vesicles (Pierre et al., 1992) and could therefore be involved in their delivery into the engorged area. The extension of the neurite becomes consolidated by bundling of microtubules in the central domain of the growth cone. Thereby, the proximal part of the growth cone resumes cylindrical shape and becomes part of the axonal shaft. Repeated courses of these three events result in axon growth. In contrast, these processes do not operate in the minor neurites and, thus, only the axon grows. Our data suggest that CLIPs have an important function during this cycle of events in the future axonal growth cone that enable neuronal polarization. By modestly stabilizing microtubules, CLIPs allow microtubules 

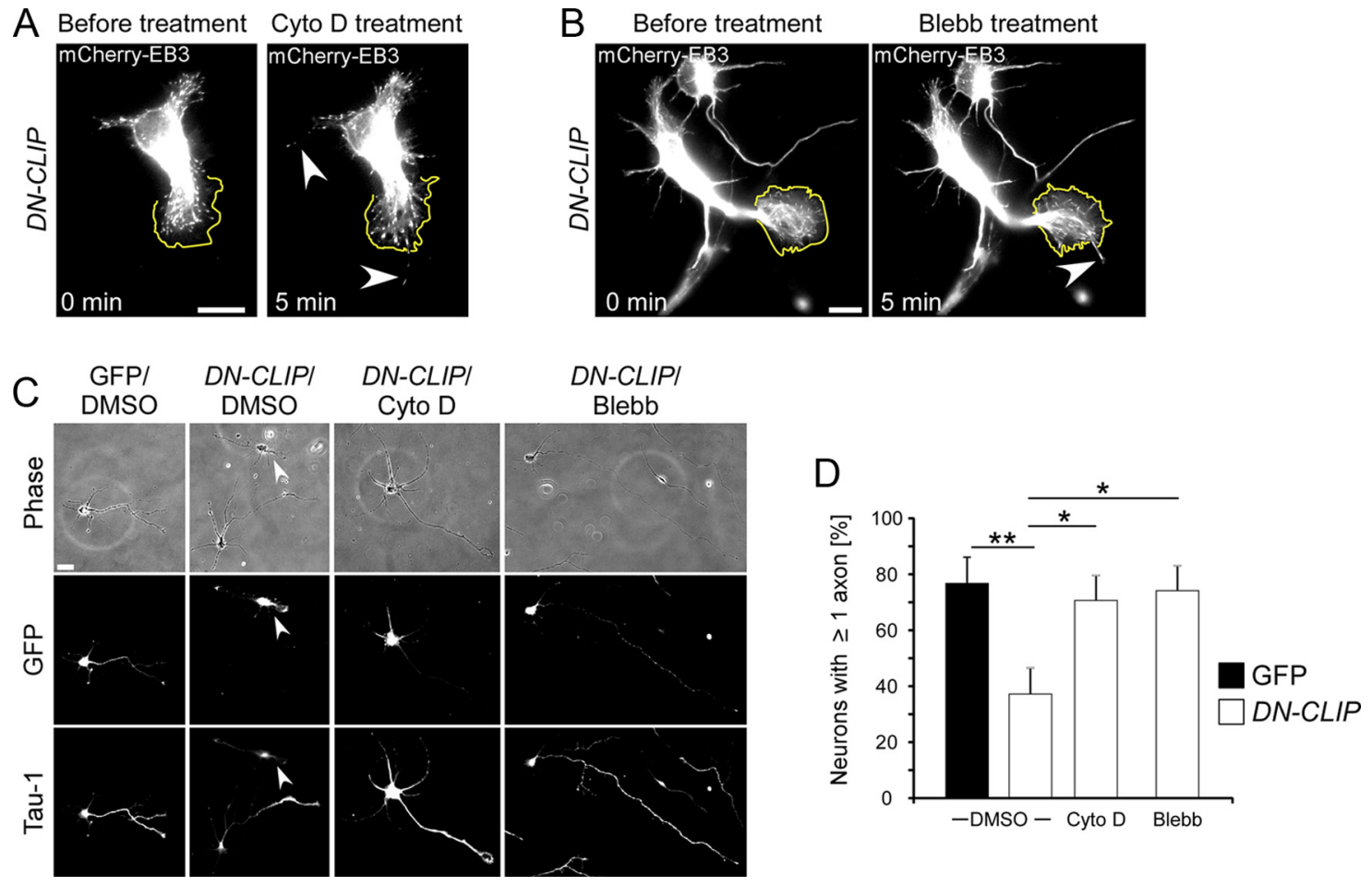

Figure 7. Depletion of the actin cytoskeleton or inhibition of myosin II rescues axon formation in DN-CLIP-transfected neurons. $A$, $B$, Neurons, double-transfected with DN-CLIP and mCherry-EB3, were treated with cytochalasin $D(C y t o D)(\boldsymbol{A})$ or blebbistatin (Blebb) (B) at 2 DIV. Five minutes after drug application EB3 comets were close to the plasma membrane (yellow line), and a thin process containing EB3 emerged out of the growth cone (arrows) ( $n=12$ neurons treated with cytochalasin D; $n=6$ for neurons treated with blebbistatin). Scale bar: $20 \mu \mathrm{m}$. C, Neurons were transfected with GFP or GFP-tagged DN-CLIP, treated with DMSO, $1 \mu \mathrm{m}$ cytochalasin D or $1 \mu \mathrm{m}$ blebbistatin, fixed at 2 DIV, and stained for the axonal marker Tau-1. Scale bar: $20 \mu \mathrm{m}$. Arrowheads indicate transfected neuron. D, Quantification of GFP-transfected (black bar) or DN-CLIP-transfected (white bars) neurons having $\geq 1$ Tau-1-positive process in cultures treated with DMSO, cytochalasin D, or blebbistatin ( $n>184$ cells from three independent cultures for each data point; ${ }^{*} p<0.05,{ }^{* *} p<0.01$ ).

to protrude to the growth cone tip. This, in turn, enables the microtubules to interact with the actin cytoskeleton, either by physically pushing against the actin network or by inducing actin-related signaling. Weakening these features by removing CLIPs from microtubules could decrease rescue frequencies. Consequently, the microtubules fail to engorge the area already protruded by actin filaments, and the growth cycle is interrupted. Instead, the splayed microtubules cannot create a corridor free of actin arcs, which is formed when microtubules are bundled (Fig. 8). As a result, the growth cone increases in size and the axon fails to extend.

How could CLIPs regulate the actin cytoskeleton in the axonal growth cone? On the one hand, CLIPs may affect intracellular signaling events. For example, in Vero cells the CLIP-170 $\mathrm{N}$ terminus directly binds IQGAP1, which, in turn, may regulate actin dynamics (Fukata et al., 2002). Conversely, the C-terminal domain of CLIP-170 binds to Lis 1 and dynactin (Coquelle et al., 2002; Goodson et al., 2003) and is thus responsible for CLIP participation in the dynein pathway. However, in this signaling-based scenario an involvement of the dynein pathway would be unlikely, as the overexpression of the CLIP

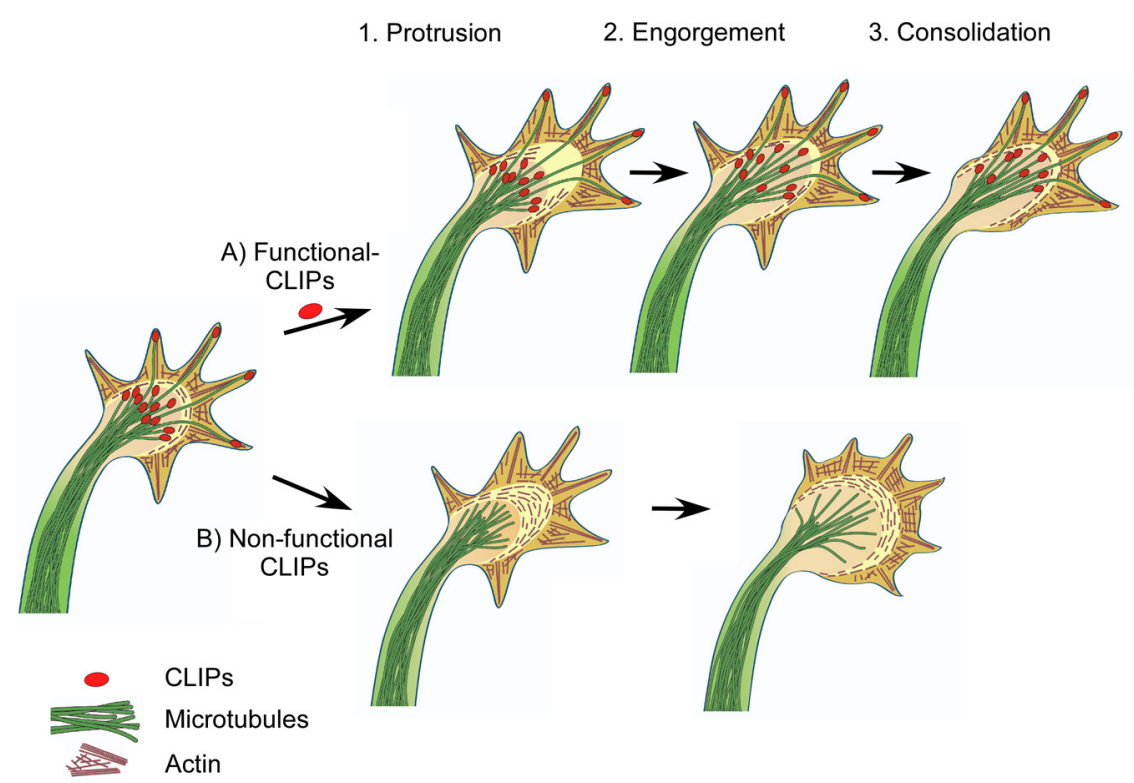

Figure 8. Model: CLIPs enable microtubule engorgement of the growth cone during axon formation. $\boldsymbol{A}$, Axon elongation consists of three repeating steps. First, filopodia and lamellipodia enable the growth cone to extend distally (Protrusion). Then, dynamic microtubules engorge from the central growth cone area into the former transitional zone, where actin filaments disassemble and the remaining arcs reorient toward the growth axis (Engorgement). Protruding microtubules transport vesicles and organelles into the engorged area. The extension of the neurite consolidates by the bundling of microtubules in the central domain; the proximal part of the growth cone resumes cylindrical shape and becomes part of the axonal shaft (Consolidation). B, Nonfunctional CLIPs lead to a decrease in microtubule stability. Hence, while filopodia and lamellipodia still protrude, microtubules fail to subsequently engorge into the actin rich area. As a result, the growth cone area increases, but the axon fails to grow. 
N-terminal domain alone was sufficient to induce axon formation. On the other hand, CLIPs could also affect the actin cytoskeleton more directly by enabling the interaction of microtubules with the actin cytoskeleton. In fact, fibroblast studies showed that microtubules and actin filaments mutually influence each other. For example, increased microtubule growth in fibroblasts after the washout of the microtubule-depolymerizing drugs nocodazole or colcemid increases actin polymerization and dynamics of lamellipodia (Bershadsky et al., 1990; WatermanStorer et al., 1999) by activating the Rho GTPase Rac1 (Waterman-Storer et al., 1999). Consistent with such interpretation, we found that modest nocodazole concentrations caused neurons with splayed microtubules at the tips, enlarged growth cones, and impaired growth cone dynamics and axon growth, recapitulating the phenotype of $D N$-CLIP-expressing neurons (data not shown). In turn, actin retrograde flow at the leading edge of fibroblasts and growth cones hinders microtubule protrusion (Waterman-Storer and Salmon, 1997; Burnette et al., 2008; Schaefer et al., 2008). Thus, microtubule dynamics affect the actin cytoskeleton and vice versa during initial neuronal polarization.

Initial neuronal polarization has been described as a "tug of war" in which each neurite aims to become the axon (Craig and Banker, 1994). In this model, an intracellular re-enhancing feedback loop singles out one neurite to become the axon, while intrinsic inhibitory cues repress the growth of the other neurites (Andersen and Bi, 2000; Witte and Bradke, 2008). The mutual influence of the actin network and the microtubule cytoskeleton could be part of this proposed positive feedback loop in the future axon. Consistently, a brief local microtubule stabilization (Witte et al., 2008) or actin depolymerization (Bradke and Dotti, 1999) bias the fate of a neurite of a yet unpolarized cell to become an axon. It suggests that a transient manipulation of either the actin or the microtubule cytoskeleton is sufficient to promote axon specification by activating reinforcing events that promote sustained axonal outgrowth. Thus, CLIPs could enable neuronal polarization by enhancing microtubule stability, which then affects growth cone dynamics and the underlying actin cytoskeleton. Changes in the actin network, in turn, can allow microtubules to protrude further distally into the growth cone (Schaefer et al., 2002, 2008), thereby generating a positive feedback loop. A reinforcement of polarized growth might be further provided by vectorial membrane and cytoplasmic flow into the future axon, which may rely on stable microtubules (Bradke and Dotti, 1997). Consistent with this possibility, specific microtubule-dependent motor proteins, including kinesin-1, transport vesicles preferentially on stable microtubules and become enriched before morphological polarization (Jacobson et al., 2006; Reed et al., 2006; Konishi and Setou, 2009). If the transported cargo contains limiting factors for axon growth, for example microtubule stabilizers including CLIPs or actin regulators such as the Wiskott-Aldrich syndrome protein (WASP)family verprolin-homologous protein (WAVE) (Kawano et al., 2005; Yokota et al., 2007; Tahirovic et al., 2010), the axon would enrich in those factors while the minor neurites would become depleted of them. Hence, CLIP-mediated stabilization of microtubules might be one entry site to induce a re-enhancing loop to form an axon.

In conclusion, we showed that CLIPs enhance microtubule stability to generate an axon. This establishes microtubule stabilization as a key process during neuronal polarization on a molecular level. Because the microtubule network and the actin cytoskeleton mutually influence each other, it implies that these cytoskeletal elements reinforce their changes to sustain axon growth. The molecular dissection of this putative enhancement loop is now the challenge ahead.

\section{References}

Andersen SS, Bi GQ (2000) Axon formation: a molecular model for the generation of neuronal polarity. Bioessays 22:172-179.

Arimura N, Kaibuchi K (2007) Neuronal polarity: from extracellular signals to intracellular mechanisms. Nat Rev Neurosci 8:194-205.

Arregui C, Busciglio J, Caceres A, Barra HS (1991) Tyrosinated and detyrosinated microtubules in axonal processes of cerebellar macroneurons grown in culture. J Neurosci Res 28:171-181.

Bai J, Ramos RL, Paramasivam M, Siddiqi F, Ackman JB, LoTurco JJ (2008) The role of DCX and LIS1 in migration through the lateral cortical stream of developing forebrain. Dev Neurosci 30:144-156.

Barnes AP, Polleux F (2009) Establishment of axon-dendrite polarity in developing neurons. Annu Rev Neurosci 32:347-381.

Bershadsky AD, Ivanova OY, Lyass LA, Pletyushkina OY, Vasiliev JM, Gelfand IM (1990) Cytoskeletal reorganizations responsible for the phorbol ester-induced formation of cytoplasmic processes: possible involvement of intermediate filaments. Proc Natl Acad Sci U S A 87:1884-1888

Bieling P, Kandels-Lewis S, Telley IA, van Dijk J, Janke C, Surrey T (2008) CLIP-170 tracks growing microtubule ends by dynamically recognizing composite EB1/tubulin-binding sites. J Cell Biol 183:1223-1233.

Binker MG, Zhao DY, Pang SJ, Harrison RE (2007) Cytoplasmic linker protein-170 enhances spreading and phagocytosis in activated macrophages by stabilizing microtubules. J Immunol 179:3780-3791.

Bradford M (1976) A rapid and sensitive method for the quantitation of microgram quantities of protein utilizing the principle of protein-dye binding. Anal Biochem 72:248-254.

Bradke F, Dotti CG (1997) Neuronal polarity: vectorial cytoplasmic flow precedes axon formation. Neuron 19:1175-1186.

Bradke F, Dotti CG (1999) The role of local actin instability in axon formation. Science 283:1931-1934.

Bradke F, Dotti CG (2000) Differentiated neurons retain the capacity to generate axons from dendrites. Curr Biol 10:1467-1470.

Brunner D, Nurse P (2000) CLIP170-like tiplp spatially organizes microtubular dynamics in fission yeast. Cell 102:695-704.

Burnette DT, Ji L, Schaefer AW, Medeiros NA, Danuser G, Forscher P (2008) Myosin II activity facilitates microtubule bundling in the neuronal growth cone neck. Dev Cell 15:163-169.

Conde C, Cáceres A (2009) Microtubule assembly, organization and dynamics in axons and dendrites. Nat Rev Neurosci 10:319-332.

Coquelle FM, Caspi M, Cordelières FP, Dompierre JP, Dujardin DL, Koifman C, Martin P, Hoogenraad CC, Akhmanova A, Galjart N, De Mey JR, Reiner O (2002) LIS1, CLIP-170's key to the dynein/dynactin pathway. Mol Cell Biol 22:3089-3102.

Craig AM, Banker G (1994) Neuronal polarity. Annu Rev Neurosci 17:267-310.

Dehmelt L, Smart FM, Ozer RS, Halpain S (2003) The role of microtubuleassociated protein $2 \mathrm{c}$ in the reorganization of microtubules and lamellipodia during neurite initiation. J Neurosci 23:9479-9490.

Dent EW, Kwiatkowski AV, Mebane LM, Philippar U, Barzik M, Rubinson DA, Gupton S, Van Veen JE, Furman C, Zhang J, Alberts AS, Mori S, Gertler FB (2007) Filopodia are required for cortical neurite initiation. Nat Cell Biol 9:1347-1359.

Fukata M, Watanabe T, Noritake J, Nakagawa M, Yamaga M, Kuroda S, Matsuura Y, Iwamatsu A, Perez F, Kaibuchi K (2002) Racl and Cdc42 capture microtubules through IQGAP1 and CLIP-170. Cell 109:873-885.

Gamblin TC, Nachmanoff K, Halpain S, Williams RC Jr (1996) Recombinant microtubule-associated protein $2 c$ reduces the dynamic instability of individual microtubules. Biochemistry 35:12576-12586.

Garvalov BK, Flynn KC, Neukirchen D, Meyn L, Teusch N, Wu X, Brakebusch C, Bamburg JR, Bradke F (2007) Cdc42 regulates cofilin during the establishment of neuronal polarity. J Neurosci 27:13117-13129.

Geraldo S, Khanzada UK, Parsons M, Chilton JK, Gordon-Weeks PR (2008) Targeting of the F-actin-binding protein drebrin by the microtubule plustip protein EB3 is required for neuritogenesis. Nat Cell Biol 10:1181-1189.

Goldberg DJ, Burmeister DW (1986) Stages in axon formation: observations of growth of Aplysia axons in culture using video-enhanced 
contrast-differential interference contrast microscopy. J Cell Biol 103:1921-1931.

Gomis-Rüth S, Wierenga CJ, Bradke F (2008) Plasticity of polarization: changing dendrites into axons in neurons integrated in neuronal circuits. Curr Biol 18:992-1000.

Goodson HV, Skube SB, Stalder R, Valetti C, Kreis TE, Morrison EE, Schroer TA (2003) CLIP-170 interacts with dynactin complex and the APCbinding protein EB1 by different mechanisms. Cell Motil Cytoskeleton 55:156-173.

Gupta KK, Paulson BA, Folker ES, Charlebois B, Hunt AJ, Goodson HV (2009) Minimal plus-end tracking unit of the cytoplasmic linker protein CLIP-170. J Biol Chem 284:6735-6742.

Hasaka TP, Myers KA, Baas PW (2004) Role of actin filaments in the axonal transport of microtubules. J Neurosci 24:11291-11301.

Hoogenraad CC, Akhmanova A, Grosveld F, De Zeeuw CI, Galjart N (2000) Functional analysis of CLIP-115 and its binding to microtubules. J Cell Sci 113:2285-2297.

Jacobs T, Causeret F, Nishimura YV, Terao M, Norman A, Hoshino M, Nikolić M (2007) Localized activation of p21-activated kinase controls neuronal polarity and morphology. J Neurosci 27:8604-8615.

Jacobson C, Schnapp B, Banker GA (2006) A change in the selective translocation of the Kinesin-1 motor domain marks the initial specification of the axon. Neuron 49:797-804.

Jaworski J, Kapitein LC, Gouveia SM, Dortland BR, Wulf PS, Grigoriev I, Camera P, Spangler SA, Di Stefano P, Demmers J, Krugers H, Defilippi P, Akhmanova A, Hoogenraad CC (2009) Dynamic microtubules regulate dendritic spine morphology and synaptic plasticity. Neuron 61:85-100.

Kawano Y, Yoshimura T, Tsuboi D, Kawabata S, Kaneko-Kawano T, Shirataki H, Takenawa T, Kaibuchi K (2005) CRMP-2 is involved in kinesin-1-dependent transport of the Sra-1/WAVE1 complex and axon formation. Mol Cell Biol 25:9920-9935.

Komarova YA, Akhmanova AS, Kojima S, Galjart N, Borisy GG (2002) Cytoplasmic linker proteins promote microtubule rescue in vivo. J Cell Biol 159:589-599.

Konishi Y, Setou M (2009) Tubulin tyrosination navigates the kinesin-1 motor domain to axons. Nat Neurosci 12:559-567.

Kunda P, Paglini G, Quiroga S, Kosik K, Caceres A (2001) Evidence for the involvement of Tiam1 in axon formation. J Neurosci 21:2361-2372.

Laemmli EK (1970) Cleavage of structural proteins during the assembly of the head of bacteriophage T4. Nature 227:680-685

Lansbergen G, Komarova Y, Modesti M, Wyman C, Hoogenraad CC, Goodson HV, Lemaitre RP, Drechsel DN, van Munster E, Gadella TW Jr, Grosveld F, Galjart N, Borisy GG, Akhmanova A (2004) Conformational changes in CLIP-170 regulate its binding to microtubules and dynactin localization. J Cell Biol 166:1003-1014.

Lee AC, Suter DM (2008) Quantitative analysis of microtubule dynamics during adhesion-mediated growth cone guidance. Dev Neurobiol 68:1363-1377.

Lee H, Engel U, Rusch J, Scherrer S, Sheard K, Van Vactor D (2004) The microtubule plus end tracking protein Orbit/MAST/CLASP acts downstream of the tyrosine kinase $\mathrm{Abl}$ in mediating axon guidance. Neuron 42:913-926.

Lowery LA, Van Vactor D (2009) The trip of the tip: understanding the growth cone machinery. Nat Rev Mol Cell Biol 10:332-343.

Martínez-López MJ, Alcántara S, Mascaró C, Pérez-Brangulí F, Ruiz-Lozano $\mathrm{P}$, Maes T, Soriano E, Buesa C (2005) Mouse neuron navigator 1, a novel microtubule-associated protein involved in neuronal migration. Mol Cell Neurosci 28:599-612.

Matenia D, Mandelkow EM (2009) The tau of MARK: a polarized view of the cytoskeleton. Trends Biochem Sci 34:332-342.
Mimori-Kiyosue Y, Grigoriev I, Lansbergen G, Sasaki H, Matsui C, Severin F, Galjart N, Grosveld F, Vorobjev I, Tsukita S, Akhmanova A (2005) CLASP1 and CLASP2 bind to EB1 and regulate microtubule plus-end dynamics at the cell cortex. J Cell Biol 168:141-153.

Muley PD, McNeill EM, Marzinke MA, Knobel KM, Barr MM, Clagett-Dame M (2008) The atRA-responsive gene neuron navigator 2 functions in neurite outgrowth and axonal elongation. Dev Neurobiol 68:1441-1453.

Niethammer P, Kronja I, Kandels-Lewis S, Rybina S, Bastiaens P, Karsenti E (2007) Discrete states of a protein interaction network govern interphase and mitotic microtubule dynamics. PLoS Biol 5:e29.

Pierre P, Scheel J, Rickard JE, Kreis TE (1992) CLIP-170 links endocytic vesicles to microtubules. Cell 70:887-900.

Reed NA, Cai D, Blasius TL, Jih GT, Meyhofer E, Gaertig J, Verhey KJ (2006) Microtubule acetylation promotes kinesin-1 binding and transport. Curr Biol 16:2166-2172.

Riedl J, Crevenna AH, Kessenbrock K, Yu JH, Neukirchen D, Bista M, Bradke F, Jenne D, Holak TA, Werb Z, Sixt M, Wedlich-Soldner R (2008) Lifeact: a versatile marker to visualize F-actin. Nat Methods 5:605-607.

Schaefer AW, Kabir N, Forscher P (2002) Filopodia and actin arcs guide the assembly and transport of two populations of microtubules with unique dynamic parameters in neuronal growth cones. J Cell Biol 158:139-152.

Schaefer AW, Schoonderwoert VT, Ji L, Mederios N, Danuser G, Forscher P (2008) Coordination of actin filament and microtubule dynamics during neurite outgrowth. Dev Cell 15:146-162.

Shi SH, Cheng T, Jan LY, Jan YN (2004) APC and GSK-3beta are involved in mPar3 targeting to the nascent axon and establishment of neuronal polarity. Curr Biol 14:2025-2032.

Stepanova T, Slemmer J, Hoogenraad CC, Lansbergen G, Dortland B, De Zeeuw CI, Grosveld F, van Cappellen G, Akhmanova A, Galjart N (2003) Visualization of microtubule growth in cultured neurons via the use of EB3-GFP (end-binding protein 3-green fluorescent protein). J Neurosci 23:2655-2664.

Tahirovic S, Hellal F, Neukirchen D, Hindges R, Garvalov BK, Flynn KC, Stradal TE, Chrostek-Grashoff A, Brakebusch C, Bradke F (2010) Racl regulates neuronal polarization through the WAVE complex. J Neurosci 30:6930-6943.

Takemura R, Okabe S, Umeyama T, Kanai Y, Cowan NJ, Hirokawa N (1992) Increased microtubule stability and alpha tubulin acetylation in cells transfected with microtubule-associated proteins MAP1B, MAP2 or tau. J Cell Sci 103:953-964.

Waterman-Storer CM, Salmon ED (1997) Actomyosin-based retrograde flow of microtubules in the lamella of migrating epithelial cells influences microtubule dynamic instability and turnover and is associated with microtubule breakage and treadmilling. J Cell Biol 139:417-434.

Waterman-Storer CM, Worthylake RA, Liu BP, Burridge K, Salmon ED (1999) Microtubule growth activates Rac1 to promote lamellipodial protrusion in fibroblasts. Nat Cell Biol 1:45-50.

Westermann S, Weber K (2003) Post-translational modifications regulate microtubule function. Nat Rev Mol Cell Biol 4:938-947.

Witte H, Bradke F (2008) The role of the cytoskeleton during neuronal polarization. Curr Opin Neurobiol 18:479-487.

Witte H, Neukirchen D, Bradke F (2008) Microtubule stabilization specifies initial neuronal polarization. J Cell Biol 180:619-632.

Yokota Y, Ring C, Cheung R, Pevny L, Anton ES (2007) Nap1-regulated neuronal cytoskeletal dynamics is essential for the final differentiation of neurons in cerebral cortex. Neuron 54:429-445.

Zhou FQ, Zhou J, Dedhar S, Wu YH, Snider WD (2004) NGF-induced axon growth is mediated by localized inactivation of GSK-3beta and functions of the microtubule plus end-binding protein APC. Neuron 42:897-912. 